\title{
Dietary Lactobacillus plantarum LS/07 and inulin in the management of chronic disease risk factors
}

\author{
Emília Hijováø, Ladislav Strojný, Izabela Bertková, Alojz Bomba and Jana Štofilová \\ Institute of Experimental Medicine, Faculty of Medicine, P. J. Šafárik University, Košice, Slovak Republic
}

The aim of this study was to investigate the possibilities of modification of chronic disease risk factors with probiotic strain Lactobacillus plantarum LS/07 and prebiotic inulin in rats with western high fat diet. The SpragueDawley rats were divided into four groups: control group (CG group), group with high fat diet (HFD group), group receiving high fat diet in combination with Lactobacillus plantarum LS/07 (HFD+PRO group), and group receiving high fat diet in combination with oligofructose enriched inulin (HFD+PRE group). The activity of $\beta$-glucuronidase, lipid parameters, bile acids, oxLDL, short chain fatty acids, and counts of coliforms and lactobacilli were determined. High fat diet as a key risk factor of chronic diseases had adverse effect on expression of metabolic and biochemical parameters. Dietary intake of Lactobacillus plantarum LS/07 (HFD+PRO group) and inulin (HFD+PRE group) suppressed weight gain of rats. In HFD+PRO group, the level of total cholesterol $(P<0.001)$, LDL-CH $(P<0.05)$, oxLDL $(P<0.001)$, total bile acids $(P<0.001)$ were statistically significantly decreased, while the production of short chain fatty acids was enhanced. Changes in the selected parameters exhibited a similar tendency also in the HFD+PRE group. Activity of $\beta$-glucuronidase was statistically significantly decreased $(P<0.001)$ in the HFD+PRE group. Lactobacillus plantarum LS/07 and inulin caused a statistically significant increase in the count of lactobacilli $(P<0.001)$ and a decrease in the number of coliforms $(P<0.001)$. These results indicate Lactobacillus plantarum LS/07 and inulin could be used in diet for human and animals as an important nutritional supplement or in medicinal products.

Key words: chronic diseases, Sprague-Dawley rats, inulin, Lactobacillus plantarum $\mathrm{LS} / 07$, prevention

Received: 25 July, 2020; revised: 08 September, 2020; accepted: 09 September, 2020; available on-line: 27 October, 2020

$\square_{\text {e-mail: hijova@pobox.sk }}$

Acknowledgements of Financial Support: This work was supported by grant VEGA 1/0372/10, project ITMS $2014^{+} 313011$ T651, and APVV-16-0176.

Abbreviations: $\beta$-GLUCUR, $\beta$-glucuronidase; HFD, high fat diet; $C G$, control group; oxLDL, oxidized low density lipoprotein; RO, probiotics; PRE, prebiotics; TBA, total bile acids; TC, total cholesterol; TG, triglyceride

\section{INTRODUCTION}

Nutrition trends and their personalization are scientific advances and show the need to adapt and develop innovations in human and veterinary medicine (Han, 2017; Tungland, 2018). Chronic diseases are a long-term interference in the patient's life. Of these, cardiovascular disease is the most common cause of death (Bomba et al., 2015). Dietary strategies that modulate the microbiota or its metabolic activity are emerging as effective tools for reducing risk factors for cardiovascular disease and indicate that indeed the way to a healthy heart may be through a healthy gut microbiota (Tuohy et al., 2014; Brusaferro et al., 2018). Many observations raise the intriguing possibility that gut microbiome modulation by prebiotics and probiotics may be the base of healthy eating pyramids recommended by regulatory agencies across the globe. The interconnection of prebiotics and probiotics with the intestinal microbiota shows that gut microbiota is identified as a possible modifiable risk factor in the development of chronic diseases (Davani-Davari et al., 2019). The manifestation of chronic diseases is the dysfunction of the intestinal microbiota. Intestinal dysbiosis adversely affects the expression of metabolic and biochemical parameters in the development of chronic diseases that affect general health.

The mechanisms of the hypolipidemic activity of probiotic bacteria are as follows: taking up and assimilation of cholesterol for stabilization of their cell membrane, binding cholesterol to cell walls of probiotics in intestine, conversion of cholesterol into coprostanol, involving deconjugation of bile acid via bile salt hydrolase catalysis, inhibition of hepatic cholesterol and triglyceride synthesis by short chain fatty acids such as propionate, and redistribution of cholesterol from plasma to the liver (Homayouni et al., 2012). The decrease in plasma cholesterol with the use of inulin could also be due to the inhibition of cholesterol synthesis by propionic acid or to the modification in bile acid metabolism (Beylot, 2005).

The aim of this study is to experimentally present the possibility of modifying the risk factors of chronic diseases with probiotic strain Lactobacillus plantarum LS/07 and prebiotic inulin in rats supplemented with high fat diet.

\section{MATERIALS AND METHODS}

Animals. Male and female Sprague-Dawley rats $(n=36)$ four months old were used in the experiment conducted in accordance with the principles of the Slovak Republic for the Care and Use of Laboratory Animals and approved by the Ethical Committee of the Faculty of Medicine of P. J. Šafárik University in Košice (Ro-1672/09-221). Rats were randomly assigned to 4 groups, 9 rats per group: CG (control group with conventional feed), HFD group with high fat diet (a high fat diet was prepared from a conventional diet supplemented with a $20 \%$ fat, Biofer Slovak Republic), HFD+PRO group (group with HFD + Lactobacillus plantarum LS/07), and HFD+PRE group (group with HFD + oligofrucose enriched inulin). Drinking water was provided ad 
libitum. Feed and water intake was monitored daily and body weights were recorded weekly. After 28 weeks of the experiment the rats were euthanized under anesthesia (Ketamine $100 \mathrm{mg} / \mathrm{kg}+$ Xylazine $15 \mathrm{mg} / \mathrm{kg}$, intraperitoneally). Blood samples were withdrawn from the heart by puncture and samples of fresh caecal digesta were also taken for further analysis.

Treatments. Treatments consisted of oligofructose enriched inulin (PRE, BeneoSynergy 1, ORAFTI, Tienen, Belgium) at the dose of $8 \% \mathrm{w} / \mathrm{w}$ of HFD. The isolation and characterization of probiotic strain Lactobacillus plantarum LS/07 was reported by Strojný and coworkers (2014). Lactobacillus plantarum LS/07 was cultured in MRS broth (Merck, Germany) prepared as night cultures at $37^{\circ} \mathrm{C}$ aerobically and provided in a dose of $3 \times 10^{9} \mathrm{CFU}$ of strain/1 mL. Then $0.5 \mathrm{~mL}$ of lactobacilli strains mixed with $9 \mathrm{~mL}$ of pasteurized milk $(0.5 \%$ fat, $20-22^{\circ} \mathrm{C}$ ) was poured into bottles and administered daily. Each rat received approximately $1.5 \times 10^{9}$ CFU lactobacilli via the oral route.

Bacteriological examination. Total counts of lactobacilli and coliform in the caecal digesta samples were determined at the end of the experiment. Caecal digesta $(1 \mathrm{~g})$ were placed into a sterile polyethylene Stomacher Lab Blenders bag (Seward, France) with $9 \mathrm{~mL}$ of sterile $0.9 \% \mathrm{NaCl}$ and mixed in a BagMixer 400 (Interscience, France). A series of 10 -fold dilutions $\left(10^{2}\right.$ to $\left.10^{8}\right)$ were made with the same sterile diluent. From each dilution, $0.1 \mathrm{~mL}$ aliquots were spread on selective McConkey agar plates (Merck, Germany) for culturing of coliforms and Rogosa agar plates (Biokar Diagnostics, France) for culturing of lactobacilli. The plates for lactobacilli culturing were maintained under anaerobic conditions (BD GasPak, Becton, Dickinson and Company, USA) and incubated at $37^{\circ} \mathrm{C}$ for $48 \mathrm{~h}$. Plates used for coliform culturing were incubated aerobically at $37^{\circ} \mathrm{C}$ for $16-18 \mathrm{~h}$. The numbers of colony forming units (CFU) are expressed as $\log 10$ CFU per gram of caecal digesta.

Measurement of caecal $\beta$-glucuronidase activity. The measurement of $\beta$-glucuronidase ( $\beta$-GLUCUR) activity in a fresh caecal digesta was described by Juskiewicz and coworkers (Juskiewicz et al., 2002). The reaction contained $0.3 \mathrm{~mL}$ substrate solution $(5 \mathrm{mM}$, Sigma Aldrich, USA) $p$-nitrophenyl- $\beta$-D-glucuronide for $\beta$-glucuronidase ( $\beta$-GLUCUR) and $0.2 \mathrm{~mL}$ of $1: 10(\mathrm{v} / \mathrm{v})$ dilution of the caecal digesta in $100 \mathrm{mM}$ phosphate buffer $(\mathrm{pH} 7.0)$. After incubation, $p$ - or 0 -nitrophenol was quantified after adding $0.25 \mathrm{M}$ cold sodium carbonate and the absorption was measured at $400 \mathrm{~nm}$. The enzymatic activity is expressed as $\mu \mathrm{mol}$ of $p$-nitrophenol per minute per gram digesta.

Biochemical analysis. Serum and plasma were separated from blood by centrifugation at $2500 \times g$ for $10 \mathrm{~min}$, and heparin was used as an anticoagulatory agent in plasma. All samples were kept frozen at $-80^{\circ} \mathrm{C}$ until further analysis. Blood serum was used to determine total bile acid levels (TBA) using a commercial kit (Trinity Biotech, Ireland). The blood plasma was used for determination of oxLDL with ELISA kit (USCN Life Science Inc., USA). Serum total cholesterol (TC), high density lipoproteins cholesterol (HDL-CH), and triglycerides (TG) levels were measured by using an automatic biochemical analytical system. Low density lipoprotein (LDL-CH) was calculated by Friedewald formula (Friedewald et al., 1972). The short chain fatty acids (SCFA) especially propionic, butyric and acetic acids were analyzed in the caecal digesta by using gas chromatography (Hewlett Packard 6890 Plus, USA). Total SCFA were expressed in $\mathrm{mmol} / 100 \mathrm{ml}$ of wet caecal digesta.

Liver cholesterol and triglycerides. After euthanasia, the liver was removed, rinsed with physiological saline solution, blotted dry with filter paper, and weighed. For extraction of liver lipids according to the method by Folch and others (Folch et al., 1957), $0.5 \mathrm{~g}$ liver tissue was ground in $10 \mathrm{~mL}$ of Folch solution (chloroform:methanol $=2: 1 / 24$ hours). The homogenate was then filtered with Whatman number 2 filter paper. The organic layer was then evaporated under a nitrogen stream. The dried lipid layer was dissolved with $1 \mathrm{~mL}$ DMSO and then used to determine the TC and TG levels by using commercial kits (Biovendor, Czech Republic).

Statistical analysis. Results are expressed as mean \pm standard deviation (S.D.). Statistical analysis was performed using analysis of variance (ANOVA) to determine the significance. Value of $P<0.05$ was considered to be statistically significant.

\section{RESULTS}

The mean body weight of rats was $320.11 \pm 67.12$ $\mathrm{g}$ at the beginning of experiment and it increased to $378.12 \pm 63.22 \mathrm{~g}$ at the end of the experiment. In the CG the mean body weight increased by $19.6 \%$, in the HFD group by $21.5 \%$, in the HFD+PRO group by $16 \%$, and in the HFD+PRE group by $15.3 \%$ at the end of the experiement, respectively. Average food consumption in the CG and the HFD groups was $18.75 \mathrm{~g} /$ day, but in $\mathrm{HFD}+\mathrm{PRO}$ group and HFD+PRE group it was 20.55 $\mathrm{g} /$ day and $19.79 \mathrm{~g} /$ day respectively. High fat diet supplemented with PRO and PRE alleviated the weight gain of rats and had an impact on metabolic activities of microbiota as compared with HFD group.

The total counts of coliforms and lactobacilli are presented in Table 1 . The caecal total counts of lactobacilli were lower in HFD group $(P<0.05)$ than in CG. Lactobacillus plantarum LS/07 and inulin attached to the high fat diet significantly reduced the count of coliforms $(P<0.001)$ and increased the count of lactobacilli $(P<0.001)$ in comparison to HFD group.

The serum cholesterol, triglyceride, HDL-CH, and LDL-CH levels are summarized in Table 2. Cholesterol and LDL-CH levels were higher in rats on high fat diet $(17 \%$ and $26 \%$ respectively) than in the CG. Compared to the HFD group, the HFD+PRO and HFD+PRE groups had significantly decreased TC values $(P<0.001)$.

Table 1. Lactobacilli and coliform total counts, $\beta$-glucuronidase activity in caecal digesta

\begin{tabular}{lllll}
\hline Parameter & CG & HFD & HFD+PRO & HFD+PRE \\
\hline$\beta$-GLUCUR $\mu \mathrm{mol} / \mathrm{min} / \mathrm{g}$ & $0.28 \pm 0.11$ & $0.41 \pm 012^{*}$ & $0.34 \pm 0.11$ & $0.12 \pm 0.06+++$ \\
\hline Lactobacilli log10CFU/g & $9.05 \pm 0.45$ & $8.65 \pm 0.26^{*}$ & $9.33 \pm 0.14+++$ & $9.28 \pm 0.29+++$ \\
\hline Coliforms log10CFU/g & $6.16 \pm 0.56$ & $6.51 \pm 0.38$ & $5.68 \pm 0.51++$ & $5.73 \pm 0.22+++$ \\
\hline
\end{tabular}

Data represent mean \pm standard deviation. Statistical significance is between ${ }^{*} \mathrm{CG} / \mathrm{HFD}$ and $+\mathrm{HFD} / \mathrm{HFD}+\mathrm{PRE}$ or $\mathrm{HFD} / \mathrm{HFD}+\mathrm{PRO}$ : $P<0.05 ;++P<0.01$; $+++P<0.001$ 
Table 2. Lipid profil changes in blood serum of experimental groups

\begin{tabular}{|c|c|c|c|c|}
\hline Parameter & $\mathrm{CG}$ & HFD & HFD+PRO & HFD+PRE \\
\hline $\mathrm{TC} \mathrm{mmol} / \mathrm{L}$ & $1.90 \pm 0.51$ & $2.31 \pm 0.65^{*}$ & $1.80 \pm 0.21^{+++}$ & $1.84 \pm 0.30^{+++}$ \\
\hline $\mathrm{LDL}-\mathrm{CH} \mathrm{mmol} / \mathrm{L}$ & $0.61 \pm 0.10$ & $0.82 \pm 0.38 *$ & $0.65 \pm 0.26^{+}$ & $0.70 \pm 0.10$ \\
\hline $\mathrm{HDL}-\mathrm{CH} \mathrm{mmol} / \mathrm{L}$ & $0.45 \pm 0.10$ & $0.50 \pm 0.17$ & $0.39 \pm 0.16$ & $0.48 \pm 0.20$ \\
\hline $\mathrm{TG} \mathrm{mmol} / \mathrm{L}$ & $1.03 \pm 0.20$ & $0.96 \pm 0.37$ & $0.88 \pm 041$ & $0.84 \pm 0.40$ \\
\hline
\end{tabular}

Data represent mean \pm standard deviation. Statistical significance is between ${ }^{*} \mathrm{CG} / \mathrm{HFD}$ and $+\mathrm{HFD} / \mathrm{HFD}+\mathrm{PRE}$ or $\mathrm{HFD} / \mathrm{HFD}+\mathrm{PRO}:{ }^{*} / P<0.05 ;+++P<0.001$

In HFD+PRO group, a significant decrease in LDL$\mathrm{CH}$ level was noted $(P<0.05)$. Levels of HDL-CH and TG were nonsignificantly decreased in HFD+PRO and HFD+PRE groups in comparison to the HFD group.

The changes in $\beta$-glucuronidase ( $\beta$-GLUCUR) activity in CG and experimental groups are summarized in Table 1. High fat diet (HFD group) increased $\beta$-GLUCUR activity $(P<0.05)$ as compared with $C G$. Lactobacillus plantarum LS/07 (HFD+PRO group) treatment nonsignificantly, and inulin (HFD+PRE group) treatment significantly reduced $(P<0.001) \beta$-GLUCUR activity in comparison to the HFD group.

Total bile acids - TBA levels and oxLDL are shown in Table 3. Increased LDL-CH in HFD corresponded with elevated levels of oxLDL. Lactobacillus plantarum LS/07 and inulin significantly $(P<0.001)$ suppressed levels of oxLDL and TBA. Hepatic lipid content (TC and TG) was higher in the HFD group than in the CG (Table 3).

Composition of SCFA in caecal digesta was reduced in the HFD group (Table 4). Lactobacillus plantarum LS/07 and inulin increased acetic acid production $(P<0.001 \mathrm{vs}$. $P<0.01)$ and butyric acid production $(P<0.05$ vs. $P<0.01)$ compared to the HFD group.

\section{DISCUSSION}

Epidemiological data indicate that nutrition is a major factor in the field of public health promotion as a preventive measure to correct disease risk. Chronic over-nutrition by unhealthy food, high in energy, high in fat and sugar, and low in natural polysaccharides is considered a key health risk factor in the development of chronic diseases, metabolic diseases, local and systemic inflam- mation (Knudsen et al., 2018; Zhang et al., 2018). The latest characterization of the human microbiome and its effect on health has led to a dramatic conceptual shift in research into the role of bioactive substances - probiotics and prebiotics in the diet. Changes in the composition of intestinal microbiota can be caused by a number of factors including changes in diet.

Probiotics, defined as microbial food supplements that beneficially affect the host by improving its intestinal microbial balance, have been used to change the composition of colonic microbiota, and selected biochemical parameters. Prebiotics are generally defined as "nondigestible food components that are resistant to digestion in the upper gastrointestinal tract, pass into the colon in the unchanged state where they must be fermented by resident large intestinal microbiota, and beneficially affect the microflora of the host organism by selectively stimulating the growth and/or activity of one or limited number of bacteria in the colon and thus improving the host health" (Gibson \& Roberfroid, 1995). Among prebiotics the most important are oligosaccharides and galactooligosaccharides, which are referred to as bifidogenic substances (bifidofactors) with reference to their ability to selectively promote the growth of Bifidobacterium spp. (B. longum, B. breve, B. pseudolongum, B. infantis, B. lactis) and Lactobacillus spp. (L. acidophilus, L. casei, L. reuteri, L. plantarum), (Hijová et al., 2019).

Prebiotics, that have not been hydrolyzed in the small intestine, become available for the microbial community in the colon. One of the important health benefits of prebiotic polysaccharides in the diet is their ability to ferment in the gut. The major end products of fermented polysaccharides produced by the gut microbiota are short chain fatty acids - SCFAs (e.g., acetate, propionate, and butyrate) and gases (e.g., $\mathrm{H}_{2}$ and $\mathrm{CO}_{2}$ ). These

Table 3. Total bile acids, ox-LDL, liver TC and liver TG in experimental groups.

\begin{tabular}{lllll}
\hline Parameter & CG & HFD & HFD+PRO & HFD+PRE \\
\hline TCA $\mu \mathrm{mol} / \mathrm{L}$ & $20.85 \pm 5.21$ & $22.38 \pm 5.20$ & $13.00 \pm 4.37^{+++}$ & $14.70 \pm 2.35^{+++}$ \\
\hline oxLDL $\mathrm{ng} / \mathrm{m} / \mathrm{L}$ & $34.70 \pm 5.00$ & $41.20 \pm 6.80^{*}$ & $28.00 \pm 1.10^{+++}$ & $30.00 \pm 2.20^{+++}$ \\
\hline liver TC $\mathrm{mg} / \mathrm{g}$ & $6.54 \pm 1.28$ & $7.33 \pm 2.58^{* *}$ & $9.98 \pm 2.99^{++}$ & $9.72 \pm 3.77^{++}$ \\
\hdashline liver TG $\mathrm{mg} / \mathrm{g}$ & $31.91 \pm 9.35$ & $48.24 \pm 9.08^{* *}$ & $21.37 \pm 5.56^{+++}$ & $31.58 \pm 9.68^{+++}$ \\
\hline
\end{tabular}

Data represent mean \pm standard deviation. Statistical significance is between ${ }^{*} \mathrm{CG} / \mathrm{HFD}$ and $+\mathrm{HFD} / \mathrm{HFD}+\mathrm{PRE}$ or $\mathrm{HFD} / \mathrm{HFD}+\mathrm{PRO}:{ }^{*} P<0.05$; $* * /++P<0.01 ;+++P<0.001$

Table 4. Composition of SCFA in caecal digesta of experimental groups

\begin{tabular}{lllll}
\hline Parameter & CG & HFD & HFD+PRO & HFD+PRE \\
\hline Acetate $\mathrm{mmol} / 100 \mathrm{~L}$ & $12.90 \pm 1.82$ & $10.81 \pm 1.58^{* * *}$ & $12.10 \pm 1.55^{+++}$ & $11.76 \pm 1.67^{++}$ \\
\hline Propionate $\mathrm{mmol} / 10 \mathrm{~L}$ & $3.32 \pm 0.33$ & $2.51 \pm 0.42^{* * *}$ & $2.64 \pm 0.42$ & $2.76 \pm 0.57^{+}$ \\
\hline Butyrate $\mathrm{mmol} / 100 \mathrm{~L}$ & $2.95 \pm 0.43$ & $2.34 \pm 0.56^{* *}$ & $2.72 \pm 0.43$ & $2.91 \pm 0.62^{+++}$ \\
\hline
\end{tabular}

Data represent mean \pm standard deviation. Statistical significance is between ${ }^{*} \mathrm{CG} / \mathrm{HFD}$ and $+\mathrm{HFD} / \mathrm{HFD}+\mathrm{PRE}$ or HFD/HFD+PRO: $+P<0.05$; $++/^{* *} P<0.01 ;+++{ }^{* * *} P<0.001$ 
reactions occur with the use of the available substrates that express a cascade of metabolic functions related to host nutrition and health benefit (Hijová \& Chmelárová, 2007; Hijová, 2019). Butyrate is produced by endogenous intestinal bacteria Faecalibacterium prausnitrii, Eubacterium rectale, Roseburia spp., Eubacterium hallii, and Ruminococcus bromii in human colon (Rios-Covián et al., 2016). Butyrate is mainly used by colonocytes as an energy source, exert strong anti-infective and anti-infammatory properties in the gut, and it is also able to prevent increases in body weight without altering food intake (Henagan et al., 2015). In our study, butyric acid production was increased in the HFD+PRO group $(P<0.05)$ and in the HFD+PRE group $(P<0.001)$ in comparison to the HFD group. It is possible, that supplementary high fat diet with PRO and PRE suppressed weight gain through butyric acid production. Propionate is dominated by relatively few bacterial genera, among which Akkermansia municiphila is the most important one (Brusaferro et al., 2018). High fat diet decreases the level of $A$. municiphila. Beneficial effect of $A$. municiphila on host physiology and microbiome composition, comes from the studies linking A. municiphila to metabolic disorders, such as diabetes and obesity (Everard et al., 2011; Ottman et al., 2017). Propionate has a protective effect in reducing the risk of cancer development and is associated with significant systemic metabolic effect, it is rapidly absorbed and can be found in the circulation, decreases serum cholesterol and liver lipogenesis (Hosseini et al., 2011). Large amount of acetic acid production can be beneficial as it is associated with the reduction of lipid accumulation in adipose tissue, protection against the accumulation of fat in the liver (Everard et al., 2014). Increased production of acetic acid in the HFD+PRO group $(P<0.001)$ and in the HFD+PRE group $(P<0.01)$ did not prevent from elevated level of total cholesterol in liver $(P<0.01)$ but had hypocholesterolemic effect in blood of rats $(P<0.001)$.

High fat diet modulates gut microbiota and plasma concentration of lipoplysaccharides, that is, metabolic endotoxemia. Our data demonstrate that high fat diet is accompanied by the significant reduction in counts of lactobacilli $(P<0.05)$. Due to these changes, eating a high fat diet (HFD group) and lower counts of lactobacilli influenced the intestinal microbiota composition and metabolic processes in the caecum content, resulting in an increased activity of $\beta$-glucuronidase $(P<0.05)$. Many studies have reported hypolipidemic and antiobesity effects of bacterial species that mainly belong to the genera Lactobacillus and Bifidobacterium and are currently of commercial interest as probiotics (Yin et al., 2010; Wang et al., 2012). The possible mechanisms of probiotic involved in the hypolipidemic effect may be as folows: a) the assimilation of cholesterol by bacterial growing cells; b) the binding of cholesterol to the bacterial cellular surface, thereby inhibiting the absorption of cholesterol back into the body; c) the deconjugation of bile acids by bacterial acid hydrolyses, inreasing cholesterol excretion of deconjugated bile salts, and increasing cholesterol uptake and metabolism in the liver as compensatory response because bile acids are synthesized from cholesterol in the liver; d) inhibition of hepatic cholesterol and triglyceride synthesis through the action of short chain fatty acids, especially propionic acid. Caecal and hepatic lipid contents were higher in high fat diet group than in the experimental groups receiving probiotic or prebiotic (HFD+PRO group, HFD+PRE group). This suggests that the hypolipidemic effect is caused by decrease of lipid absorption because of the Lactobacillus plantarum LS/07 and increase in lipid catabolism because of inulin.
These data show that administration of Lactobacillus plantarum LS/07 and inulin plays an important role in the treatment of risk factors for chronic diseases. Action of probiotics and prebiotics through the modulation of intestinal microbiota point out that gut microbiota can be considered as a possible modifiable risk factor in the development of chronic diseases.

\section{REFERENCES}

Beylot M (2005) Effects of inulin-type fructans on lipid metabolism in man and in animal models. BJN 93: S163-S168. https://doi. org/10.1079/BJN20041339

Bomba A, Strojný L, Salaj R, Štofilová J (2015) Modulation of gut microflora in the prevention of atherosclerosis and cancer using probiotics and prebiotics. In Beneficial Microbes in Fermented and Functional Foods, pp 413-435. Boca Raton: Taylor \& Francis Group. ISBN 978-1 4822-0662-3

Brusaferro A, Cozzali R., Orabona C, Biscarini A, Farinelli E, Cavalli E, Grohmann U, Principi N, Esposito S (2018) Is it time to use probiotics to prevent or treat obesity? Nutrients 10: 1613. https:// doi.org/10.3390/nu10111613

Davani-Davari D, Negahdaripour M, Karimzadeh I, Seifan M, Mohkam M, Masoumi SJ, Berenjian A, Ghasemi Y (2019) Prebiotics: Definition, types, sources, mechanisms, and clinical applications. Foods 8: 92. https://doi.org/10.3390/foods 8030092

Everard A, Lazarevic V, Derrien M, Girard M, Muccioli GG, Neyrinck M, Possemiers S, Holle AV, Francois P, Vos WM, Delzenne NM, Schrenzel J, Cani PD (2011) Responses of gut microbiota and glucose and lipid metabolism to prebiotics in genetic obese and dietinduced leptin resistant mice. Diabetes 60: 2775-2786. https://doi. org/10.2337/db11-0227

Everard A, Lazarevic V, Gaïa N, Johansson M, Ståhlman M, Backhed F, Delzenne NM, Schrenzel J, François P, Cani PD (2014) Microbiome of prebiotic- treated mice revels novel targets involved in host response during obesity. ISME J 8: 2116-2130. https://doi. org/10.1038/ismej.2014.45

Folch JM, Lees M, Solane SGH (1957) A simple method for the isolation and purification of total lipides from animal tissues. JBC 226: 497-509

Friedewald WT, Levy RI, Fredrickson DS (1972) Estimation of the concentration of low-density lipoprotein cholesterol in plasma, without use of the preparative ultracentrifuge. Clin Chem 18: 499-502

Gibson GR, Roberfroid MB (1995) Dietary modulation of the human colonic microbiota. Introducing the concept of prebiotics. J Nutr 125: 1401-1412. https://doi.org/10.1093/in/125.6.1401

Han KH, Yamamoto A, Shimada K, Kikuchi H, Fukushima M (2017) Dietary fat content modulates the hypolipidemic effect of dietary inulin in rats. Mol Nutr Food Res 61: 1600635. https://doi. org/10.1002/mnfr. 201600635

Henagan TM, Stefanska B, Fang Z, Navard AM, Ye J, Lenard NR, Devarshi PP (2015) Sodium butyrate epigenetically modulates highfat diet-induced skeletal muscle mitochondrial adaptation, obesity and insulin resistance through nucleosome positioning. Br J Pharmacol 172: 2782-2798. https://doi.org/10.1111/bph.13058

Hijová E and Chmelárová A (2007) Short chain fatty acids and colonic health. Bratisl Lek List 108: 354-358.

Hijová E, Bertková I, Stofilová J (2019) Dietary fibre as prebiotics in nutrition. Cent Eur J Public Health 27: 251-255. https://doi. org/10.21101/cejph.a5313.

Hijová E (2019) Gut bacterial metabolites of indigestible polysaccharides in intestinal fermentation as mediators of public health. Bratisl Med J 120: 807-812. https://doi.org/10.4149/BLL 2019134

Homayouni A, Payahoo L, Azizi A (2012) Effects of probiotics on lipid profile: a review. Am J Food Technol 7: 251-265. https://doi. org/10.3923/ajft.2012.251.265

Hosseini E, Grootaert C, Verstraete W, Van de Wiele T (2011) Propionate as a health-promoting microbial metabolite in the human gut. Nutr Rev 69: 245-258. https://doi.org/10.1111/j.1754887.2011.00388.x

Juskiewicz J, Zdunczyk Z, Wroblewska M, Oszmianski J, Hernandez $\mathrm{T}$ (2002) The responce of rats to feeding with diets containing grapefruit flavonoid extract. Food Res Int 35: 201-205. https://doi. org/10.1016/S0963-9969(01)00184-3

Knudsen KEB, Lærke HN, Hedemann MS, Nielsen TS, Ingerslev AK, Nielsen DSG, Theil PK, Purup S, Hald S, Schioldan AG, Marco ML, Gregersen S, Hermanse K (2018) Impact of diet-modulated butyrate production on intestinal barrier function and inflammation. Nutrients 10: 1499. https://doi.org/10.3390/nu10101499

Ottman N, Geerlings SY, Aalvink S, Vos WM, Belzer C (2017) Action and function of Akkermansia municiphila in microbiome ecology, health and diseases. Best Practice \& Res Clin Gastroenterol 31: 637-642. https://doi.org/10.1016/j.bpg.2017.10.001 
Rios-Covián D, Ruas-Madiedo P, Margolles A, Gueimonde M, de Los Reyes-Gavilán CG, Salazar N (2016) Intestinal short chain fatty acids and their link with diet and human health. Front Microbiol 7: 185. https://doi.org/10.3389/frmicb.2016.00185

Strojný L, Štofilová J, Hijová E, Szabadosova V, Salaj R, Bertková I, Chmelárová A, Čokášová D, Pramuková B, Brandeburova A, Bomba A, Bobrov N, Suchánek P (2014) Effect of Lactobacillus plantarum LS/07 in combination with flaxseed oil on the microflora, enzymatic activity and histological changes in the development of chemically induced precancerous growth in the colon. Czech J Anim Sci 59: 268-277. https://doi.org/10.17221/7497-CJAS

Tungland B (2018) Human Microbiota in Health and Disease: From Pathogenesis to Therapy. pp 649. Elsevier Inc. ISBN 9780128146507

Tuohy KM, Fava F, Viola R (2014) The way to a man's heart is through his gut microbiota-dietary pro- and prebiotics for the man- agement of cardiovascular risk. Proc Nutr Soc 72: 172-185. https:// doi.org/10.1017/S0029665113003911

Wang J, Zhang H, Chen X, Chen Y, Menghebilige M, Bao Q (2012) Selection of potential probiotic lactobacilli for cholesterol-lowering properties and their effect on cholesterol metabolism in rats fed a high-lipid diet. J Dairy Sci 95: 1645-1654. https://doi. org/10.3168/jds.2011-4768

Yin YN, Yu, QF, Fu N, Liu XW, Lu FG (2010) Effects of four Bifidobacteria on obesity in high-fat diet induced rats. World J Gastroenterol 16: 3394-3401. https://doi.org/10.3748/wjg.v16.i27.3394

Zhang T, Yang Y, Liang Y, Jiao X, Zhao Ch (2018) Beneficial effect of intestinal fermentation of natural polysaccharides. Nutrients 10 : 1055. https://doi.org/10.3390/nu10081055 